\title{
A revised view of cardiac sodium channel "blockade" in the long-QT syndrome
}

\author{
Nicholas G. Kambouris, ${ }^{1}$ H. Bradley Nuss, ${ }^{2}$ David C. Johns, ${ }^{2}$ Eduardo Marbán, ${ }^{2}$ \\ Gordon F. Tomaselli, ${ }^{2}$ and Jeffrey R. Balser ${ }^{3}$ \\ ${ }^{1}$ Department of Anesthesiology, and \\ ${ }^{2}$ The Institute of Molecular Cardiobiology, The Johns Hopkins University School of Medicine, \\ Baltimore, Maryland 21205, USA \\ ${ }^{3}$ Departments of Anesthesiology and Pharmacology, Vanderbilt University School of Medicine, \\ Nashville, Tennessee 37232, USA \\ Address correspondence to: Jeffrey R. Balser, Room 560, MRB II, Vanderbilt University School of Medicine, Nashville, \\ Tennessee 37232, USA. Phone: (615) 936-0277; Fax: (615) 936-0456; E-mail: jeff.balser@mcmail.vanderbilt.edu.
}

Received for publication December 21, 1999, and accepted in revised form March 2, 2000.

\begin{abstract}
Mutations in SCN5A, encoding the cardiac sodium (Na) channel, are linked to a form of the congenital long-QT syndrome (LQT3) that provokes lethal ventricular arrhythmias. These autosomal dominant mutations disrupt $\mathrm{Na}$ channel function, inhibiting channel inactivation, thereby causing a sustained ionic current that delays cardiac repolarization. Sodium channel-blocking antiarrhythmics, such as lidocaine, potently inhibit this pathologic Na current $\left(I_{\mathrm{Na}}\right)$ and are being evaluated in patients with LQT3. The mechanism underlying this effect is unknown, although high-affinity "block" of the open Na channel pore has been proposed. Here we report that a recently identified LQT3 mutation (R1623Q) imparts unusual lidocaine sensitivity to the Na channel that is attributable to its altered functional behavior. Studies of lidocaine on individual R1623Q single-channel openings indicate that the open-time distribution is not changed, indicating the drug does not block the open pore as proposed previously. Rather, the mutant channels have a propensity to inactivate without ever opening ("closed-state inactivation"), and lidocaine augments this gating behavior. An allosteric gating model incorporating closed-state inactivation recapitulates the effects of lidocaine on pathologic $\mathrm{I}_{\mathrm{Na}}$. These findings explain the unusual drug sensitivity of R1623Q and provide a general and unanticipated mechanism for understanding how Na channel-blocking agents may suppress the pathologic, sustained Na current induced by LQT3 mutations.
\end{abstract}

J. Clin. Invest. 105:1133-1140 (2000).

\section{Introduction}

Although our understanding of the drug targets and therapeutic options for cardiac arrhythmias has expanded over the last decade, insight into the functional mechanism of antiarrhythmic drug action is incomplete, and the pharmacologic management of arrhythmias remains plagued by poor efficacy and unacceptable toxicity. Sodium (Na) channel "blockers" are widely used to treat all forms of life-threatening cardiac arrhythmias but may be particularly useful in managing patients with autosomal dominant forms of the long-QT syndrome that involve mutations in the cardiac Na channel gene SCN5A (LQT3) (1). An improved understanding of the molecular pharmacology of $\mathrm{Na}$ channel blockers in this relatively rare inherited disorder may advance the clinical management of these patients while improving our understanding of drug action in more common, acquired forms of sudden cardiac death.

Cardiac Na channels initiate excitation in the atria and ventricles, but normally open only briefly, fully inactivating within a few milliseconds. The LQT3 mutations involve amino acid residues that reside in domains critical to the inactivating function of $\mathrm{Na}$ channels (in the III-IV interdomain linker [2,3] and vicinal residues
[4-8]). Hence, these mutations destabilize inactivation and sustain the $\mathrm{Na}$ current $\left(I_{\mathrm{Na}}\right)$ during the action potential plateau $(9-11)$. This pathologic, sustained $I_{\mathrm{Na}}$ delays cellular repolarization, prolonging the electrocardiographic QT interval and predisposing patients to polymorphic ventricular tachycardia. Lidocaine binds to amino acids positioned on the intracellular side of the aqueous, ion-conducting pore (12-14), near (and perhaps overlapping) the inactivation gating domains (15). It is apparent that lidocaine and its analogues reduce the pathologic current through $\mathrm{Na}$ channels with SCN5A mutations $(10,16)$, and this may underlie the beneficial clinical effect of these agents in patients with LQT3 disorders (17). However, the molecular basis for this pharmacologic effect remains unclear.

Suppression of the pathologic Na current might involve a high-affinity interaction between lidocaine and the open conducting state of the Na channel (18) whereby the drug essentially "plugs" the open pore. Alternatively, the drugs might somehow enhance or "repair" the disordered inactivation gating function $(19,20)$. Recently, a sporadic missense mutation in the voltage-sensing region of the cardiac $\mathrm{Na}$ channel (the S4 segment of domain IV; R1623Q) was reported in a 
Japanese girl who had the long-QT syndrome and who was effectively treated with mexiletine, a lidocaine analogue (21). Heterologous expression of human heart $\mathrm{Na}$ channels (hH1) with R1623Q revealed destabilized inactivation from the open state $(22,23)$ consistent with the important role of the domain IV-S4 charge sensor in inactivation gating $(24,25)$. In addition, it appeared that this disrupted inactivation phenotype, in analogy to the other LQT3 disorders, was at least partly corrected by lidocaine (22).

Here we report that the R1623Q Na channel is unusually sensitive to lidocaine. Surprisingly, we find that lidocaine neither plugs the open channel, nor repairs the inactivation of open channels as proposed previously (22). Rather, our findings reveal an unanticipated mechanism for lidocaine action. We find that lidocaine augments an intrinsic inactivation gating process that is amplified in R1623Q, known as closed-state inactivation, and thereby prevents channel opening altogether. Our results reveal a molecular mechanism for the unusual lidocaine sensitivity of this particular mutant, while implicating closed-state inactivation as an important functional therapeutic target for $\mathrm{Na}$ channel-blocking agents in other long QT disorders.

\section{Methods}

Site-directed mutagenesis of residue $\mathrm{R} 1623$ in the hH1 $\mathrm{Na}$ channel $\alpha$ subunit was performed using standard methods (26) and was sequence verified. For expression in Xenopus oocytes, $\alpha$-subunit cRNAs were coinjected with an equimolar ratio of $\beta_{1}$ subunit cRNA as described previously (22). For study in mammalian cells, both wildtype and R1623Q full-length $\alpha$-subunit cDNAs were subcloned from the host vector pSP64T into the HindIII-XbaI site of vector PGFPIRS for bicistronic expression of the channel and GFP reporter proteins (27). Stable cell lines were generated by first linearizing the plasmids at the AseI site. Linearized DNA was transfected into HEK293 cells (CRL-1573, American Type Culture Collection, Manassas, Virginia, USA) using Lipofectamine (Life Technologies, Rockville, Maryland, USA) as direct-

\section{Figure 1}

R1623Q channels exhibit enhanced sensitivity to lidocaine. (a) Wholecell Na currents in Xenopus oocytes depolarized from -100 to $-20 \mathrm{mV}$ in the presence (dotted line) and absence (solid line) of $200 \mu \mathrm{M}$ lidocaine. For both wild-type (right panel) and R1623Q (left panel), paired observations from a single oocyte are shown. (b) Summarized $\tau_{50}$ data before (open bars) and after (solid bars) exposure to $200 \mu \mathrm{M}$ lidocaine. $\tau_{50}$ represents the time $(\mathrm{ms})$ from peak $/_{\mathrm{Na}}$ to $50 \%$ decay (R1623Q control, $n=17$; R1623Q $200 \mu \mathrm{M}$ lidocaine, $n=14$; wildtype control, $n=9$; wild-type $200 \mu \mathrm{M}$ lidocaine, $n=9$ ). For R1623Q, $\tau_{50}$ was significantly reduced by lidocaine $\left({ }^{A} P<0.01\right)$. (c) Summary of paired data comparing the fractional reduction of peak $I_{\mathrm{Na}}$ in the presence versus the absence of $200 \mu \mathrm{M}$ lidocaine for oocytes expressing either R1623Q (left bar, $n=14$ ) or wild-type (right bar, $n=9$ ). Lidocaine suppression of peak $I_{\mathrm{Na}}$ was greater for R1623Q $\left({ }^{A} P<0.05\right)$. ed by the manufacturer. Forty-eight hours after transfection, cells were cultured in DMEM (CellGro; Mediatech, Washington, DC, USA) supplemented with $10 \%$ FBS, $2 \mathrm{mM}$ Glutamax, $1 \times$ penicillin-streptomycin, 15 mM HEPES, $\mathrm{pH} 7.4$, and $500 \mu \mathrm{g} / \mathrm{mL}$ geneticin sulfate (G 418; Life Technologies) at $37^{\circ} \mathrm{C}$ in a $5 \% \mathrm{CO}_{2}$-humidified incubator. After 1-2 weeks of selection, single colonies were isolated based on their level of fluorescence and examined by patch-clamp analysis. Desirable clones were maintained in selection media for further analysis.

Whole-cell Na currents $\left(I_{\mathrm{Na}}\right)$ were recorded from oocytes with a 2-electrode voltage clamp using ND-96 solution containing (in mM): $96 \mathrm{NaCl}, 2 \mathrm{KCl}, 1 \mathrm{MgCl}_{2}, 5$ HEPES, $\mathrm{pH}$ 7.6. $I_{\mathrm{Na}}$ was recorded from HEK cells expressing either wild-type or R1623Q Na channels at room temperature using the whole-cell configuration of the patch clamp (28) (Axopatch 200B; Axon Instruments, Burlingame, California, USA). External solutions were (in mM): 140 $\mathrm{NaCl}, 5.4 \mathrm{KCl}, 10$ glucose, $1 \mathrm{MgCl}_{2}, 0.1 \mathrm{CaCl}_{2}, 10$ HEPES, $\mathrm{pH}$ 7.4. Glass pipettes contained (in $\mathrm{mM}$ ): $140 \mathrm{KCl}, 1$ $\mathrm{MgCl}_{2}, 4 \mathrm{MgATP}, 10$ HEPES, $5 \mathrm{NaCl}, 5$ EGTA, pH 7.4; $60-80 \%$ of the series resistance $(\sim 6 \mathrm{M} \Omega)$ was compensated. For cell-attached single-channel recordings, a bath solution was used to bring the membrane potential to zero; the bath contained (in $\mathrm{mM}$ ): $140 \mathrm{KCl}, 10 \mathrm{HEPES}$, $\mathrm{pH}$ 7.4. Pipettes were filled with a recording solution containing (in $\mathrm{mM}$ ): $140 \mathrm{NaCl}, 10$ HEPES, $\mathrm{pH}$ 7.4. Singlechannel currents were filtered at $2 \mathrm{kHz}$, sampled every 50 microseconds, and were idealized using a half-height criterion with 3-point detection. Lidocaine $\mathrm{HCl}$ (1.5\% preservative-free; Abbott Laboratories, Abbott Park, Illinois, USA) was added to the patch pipette (single channels) or the bath solution (whole-cell currents) in appropriate amounts to give the concentrations indicated in each figure legend. In all cases, data were recorded after a 5minute equilibration period.

Pooled data are expressed as means \pm SD, and statistical comparisons were made using 1-way ANOVA. Exponential or Boltzmann functions were fitted to the data using nonlinear least-squares methods (Microcal Origin; Microcal Inc., Northhampton, Massachusetts,

a
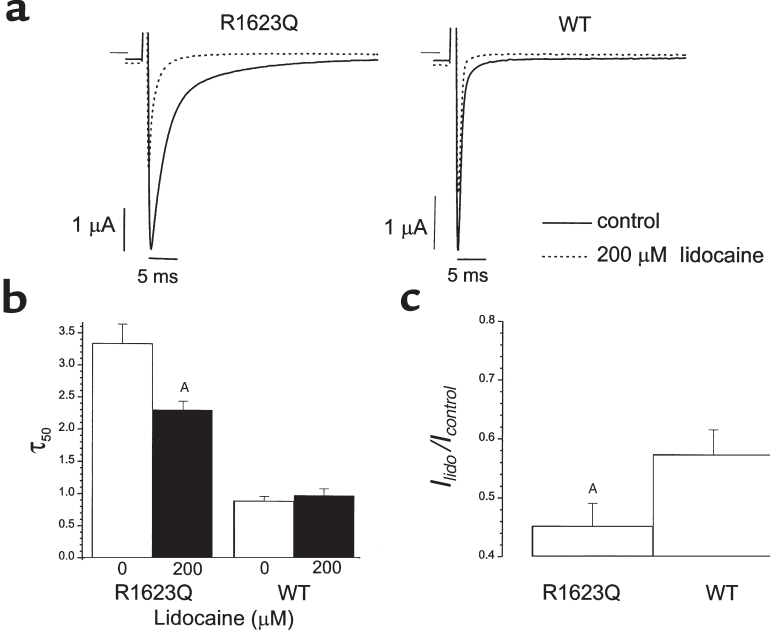

C

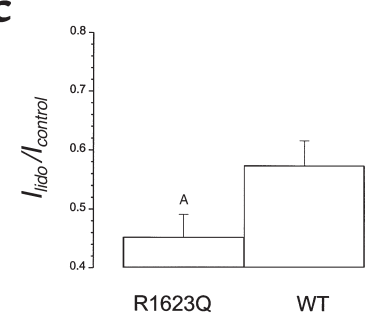


USA). The sensitivities of Markov gating models to lidocaine block (see Figure 7) were examined using numerical integration methods tailored for stiff sets of differential equations $(29,30)$ and linked to customwritten software as described previously (31).

\section{Results}

Lidocaine effects on whole oocyte R1623Q currents. To examine the general features of R1623Q gating and lidocaine block, we examined whole oocyte $I_{\mathrm{Na}}$ (Figure 1). Wildtype (Figure 1a, part i) and R1623Q (Figure 1a, part ii) $I_{\mathrm{Na}}$ is shown before and during exposure to $200 \mu \mathrm{M}$ lidocaine. As shown previously, a major feature of the drug-free R1623Q phenotype is slowed decay of $I_{\mathrm{Na}}(22$, 23), a feature that differs from LQT3 mutations in or near the III-IV linker $(10,11,15)$. Figure 1a illustrates that lidocaine has little effect on the rate of wild-type $I_{\mathrm{Na}}$ decay, but speeds the decay of R1623Q $I_{\mathrm{Na}}$, partly correcting the abnormal phenotype (22). The $I_{\mathrm{Na}}$ decay rate was quantified using a model-independent index: the time from peak current to $50 \%$ decay $\left(\tau_{50}\right)$. The summary data demonstrate that lidocaine not only speeds the rate of R1623Q $I_{\mathrm{Na}}$ decay (Figure $1 \mathrm{~b},{ }^{\mathrm{A}} P<0.01$ ), but also blocks R1623Q peak $I_{\mathrm{Na}}$ more potently than wild-type (Figure $1 c,{ }^{A} P<0.05$ ). To identify a unifying mechanism for these distinct lidocaine effects, we examined $\mathrm{Na}$ channel gating more precisely using whole-cell and cellattached patch recordings in cultured mammalian cells. Lidocaine effects on whole-cell R1623Q current in HEK-293 cells. Figure $2 \mathrm{a}$ shows wild-type (left) and R1623Q (right) $I_{\mathrm{Na}}$ in the absence (top) and presence (bottom) of $200 \mu \mathrm{M}$ lidocaine. Consistent with the whole-oocyte data, the phenotype of R1623Q in cultured cells included a slowed rate of $I_{\mathrm{Na}}$ decay. Furthermore, lidocaine had a small effect on wild-type $I_{\mathrm{Na}}$ decay, but markedly speeded the decay of R1623Q $I_{\mathrm{Na}}$. Figure $2 \mathrm{~b}$ quantifies the effects of lidocaine on $I_{\mathrm{Na}}$ decay $\left(\tau_{50}\right)$ over a range of membrane potentials. Intriguingly, the extent to which lidocaine sped the decay rate was relatively independent of voltage (change in mean $\tau_{50}$ was $38 \%$ at $-30 \mathrm{mV}$ vs. $33 \%$ at $+20 \mathrm{mV}$ ), despite a significant voltage dependence in the rate of $I_{\mathrm{Na}}$ decay. At a more physiologically relevant lidocaine concentration (20 $\mu \mathrm{M})$, R1623Q $\tau_{50}$ at $-20 \mathrm{mV}$ was still reduced to $2.7 \pm$ 0.8 milliseconds $(n=7$, not shown) compared with control ( $4.5 \pm 0.2$ milliseconds; $n=18, P<0.001)$.

The surprising lack of voltage-dependence in the blocking effect in Figure $2 \mathrm{~b}$ led us to speculate that lidocaine influences R1623Q current decay through a mechanism distinct from open-state block. The cardiac $\mathrm{Na}$ channel is incompletely available at typical "resting" membrane potentials $(-100 \mathrm{mV}$ to $-90 \mathrm{mV}$ ) owing to a substantial degree of inactivation from closed (preopen) states (32). We considered whether closed-state inactivation is enhanced by the R1623Q mutation and, if so, whether this altered gating behavior is the basis of the augmented lidocaine sensitivity of the mutant channel. The voltage-dependent availability of wildtype and R1623Q channels was examined over a range of membrane potentials negative to the channel-opening threshold (Figure 3 , inset). Figure 3 a shows peak $I_{\mathrm{Na}}$ plotted as a function of the conditioning voltage (normalized to the $-120 \mathrm{mV}$ measurement). The solid curves represent nonlinear least-squares fits of a Boltzmann function to the data (see Figure 3 legend). R1623Q $V_{1 / 2}$ was shifted leftward relative to wild-type $(-70.0 \pm 2.2 \mathrm{mV}$ vs. $-62.8 \pm 1.2 \mathrm{mV}, P=0.0071)$, and the slope factor was also increased ( $7.96 \pm 0.6$ vs. $5.55 \pm 0.4$, $P=0.0015)$. These changes both reflect reduced channel availability at membrane potentials negative to the activation threshold $(\sim-50 \mathrm{mV})$, consistent with increased inactivation from closed states.
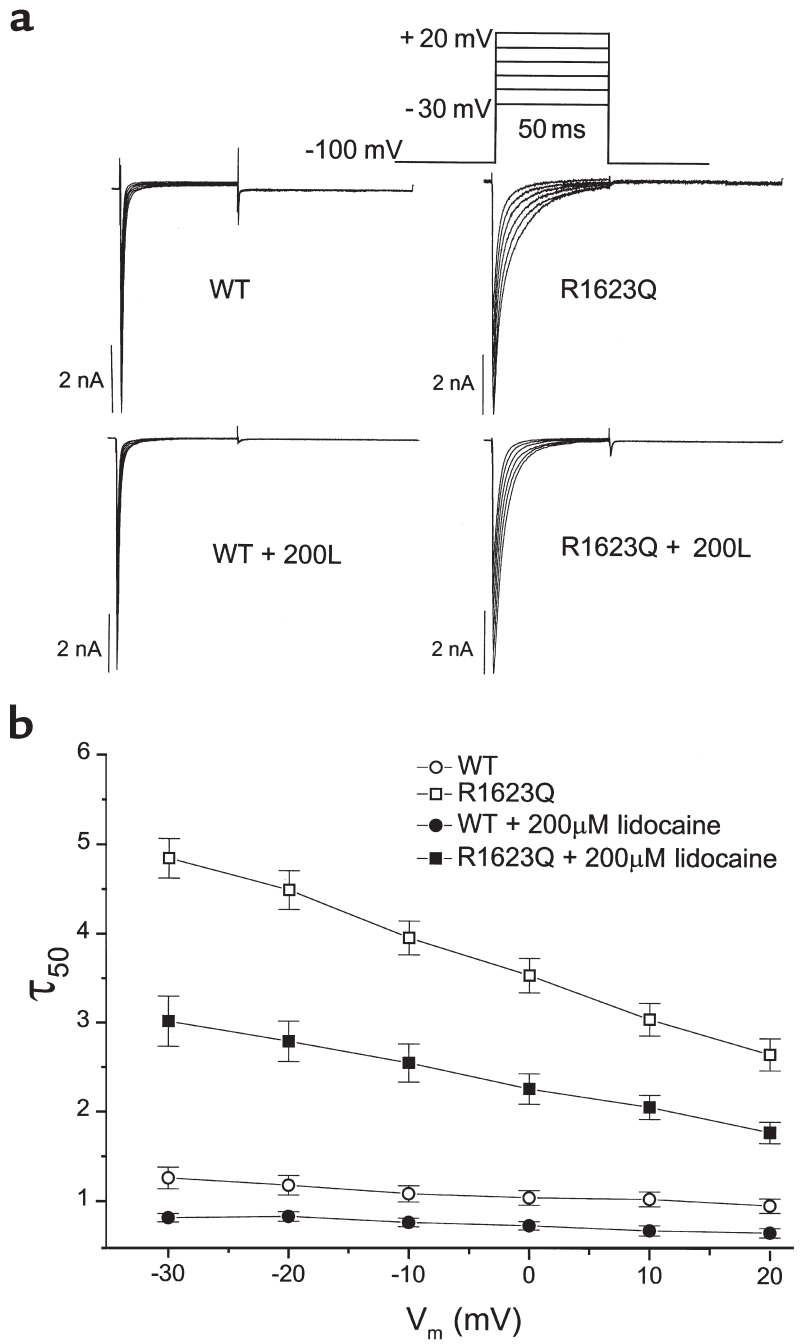

\section{Figure 2}

Lidocaine action on R1623Q currents expressed in HEK cells. (a) Family of $\mathrm{Na}$ currents elicited from cells stably expressing either wildtype (WT: left panels) or R1623Q (right panels) in the absence (top) or presence (bottom) of $200 \mu \mathrm{M}$ lidocaine. The voltage clamp protocol used is shown in the inset; a recovery interval of 20 seconds at $-100 \mathrm{mV}$ was interposed between successive step depolarizations for all experiments. (b) Decay time $\left(\tau_{50}\right)$ from peak inward $/_{\mathrm{Na}}$ to $50 \%$ decay. As shown, $200 \mu \mathrm{M}$ lidocaine hastened decay times at all voltages tested for both WT (closed circles, $n=10$ ) and R1623Q (closed squares, $n=14$ ) when compared with the drug-free condition for WT (open circles, $n=19$ ) and R1623Q (open squares, $n=18$ ). 
Voltage-dependent availability was then assessed in either 20 or $200 \mu \mathrm{M}$ lidocaine using the same voltageclamp protocol (Figure 3b). Both wild-type and R1623Q channels exhibited a dose-dependent hyperpolarizing shift in the voltage dependence of channel availability. Solid lines show least-squares fits of a Boltzmann function to the data (see legends for all fitted parameters). The effect of $200 \mu \mathrm{M}$ lidocaine on the R1623Q slope factor was especially profound $(9.31 \pm 0.45)$, reflecting a substantial loss of availability at $-100 \mathrm{mV}$, consistent with the enhanced tonic block sensitivity noted for the mutant channel in Figure 1c. Thus, lidocaine blockade augments the effect of the R1623Q mutation to reduce channel availability at membrane potentials below the opening threshold, suggesting that the altered closedstate inactivation gating and lidocaine sensitivity of the mutant may be functionally linked.

We postulated that an interaction between closedstate inactivation and lidocaine should alter the kinetics of recovery from depolarization-induced block (that is, use-dependent block). In Figure 4, recovery of availability for wild-type and R1623Q was assessed at -80 $\mathrm{mV}$, a membrane potential where R1623Q channels undergo more closed-state inactivation (Figure $3 \mathrm{a}$ ). In control and drug-exposed $(20 \mu \mathrm{M})$ conditions, both wild-type and mutant channels exhibited biexponential recovery kinetics (solid lines, Figure 4) characterized by a prominent rapid component $(\tau \sim 10-20 \mathrm{~ms})$ and a small slow component $(\tau \sim 80-150$ ms; see Figure 4 legend for fitted parameters). Consistent with previous studies (15), $20 \mu \mathrm{M}$ lidocaine did not delay the recovery of the wild-type channels following a 50-millisecond depolarizing pulse (Figure 4a). In contrast, this low concentration of lidocaine significantly delayed recovery of R1623Q channels (Figure 4b), increasing the amplitude of the slow component from 18 to $36 \%$. In similar experiments that measured recovery at -100
$\mathrm{mV}, 20 \mu \mathrm{M}$ lidocaine did not slow either wild-type or R1623Q recovery (data not shown), consistent with the minimal steady-state block of R1623Q at more hyperpolarized membrane potentials (see Figure $3 b$ ).

Effects of lidocaine on unitary R1623Q currents. We used single-channel recording to distinguish between enhanced closed-state inactivation and open-state block as the basis of the augmented lidocaine sensitivity of R1623Q. Figure 5 shows unitary current records from HEK-293 cells stably expressing wild-type (left) and R1623Q channels (right) in the absence or presence of $200 \mu \mathrm{M}$ lidocaine. Inspection of the current records reveals that the R1623Q mutation increased the number of openings during each depolarization and also lengthened the duration of the openings $(22,23)$. The current records suggest lidocaine reduced the overall number of openings, but had little effect on the character of individual openings.

To analyze these effects quantitatively, we first examined the mean open time. Figure 5 (bottom) shows representative histograms of open-time distributions at $-20 \mathrm{mV}$ for wild-type (left) and R1623Q (right) channels in the absence and presence of $200 \mu \mathrm{M}$ lidocaine. Because wild-type and R1623Q channels both exhibited 2 open-time components, a biexponential function was fitted to the histograms (superimposed

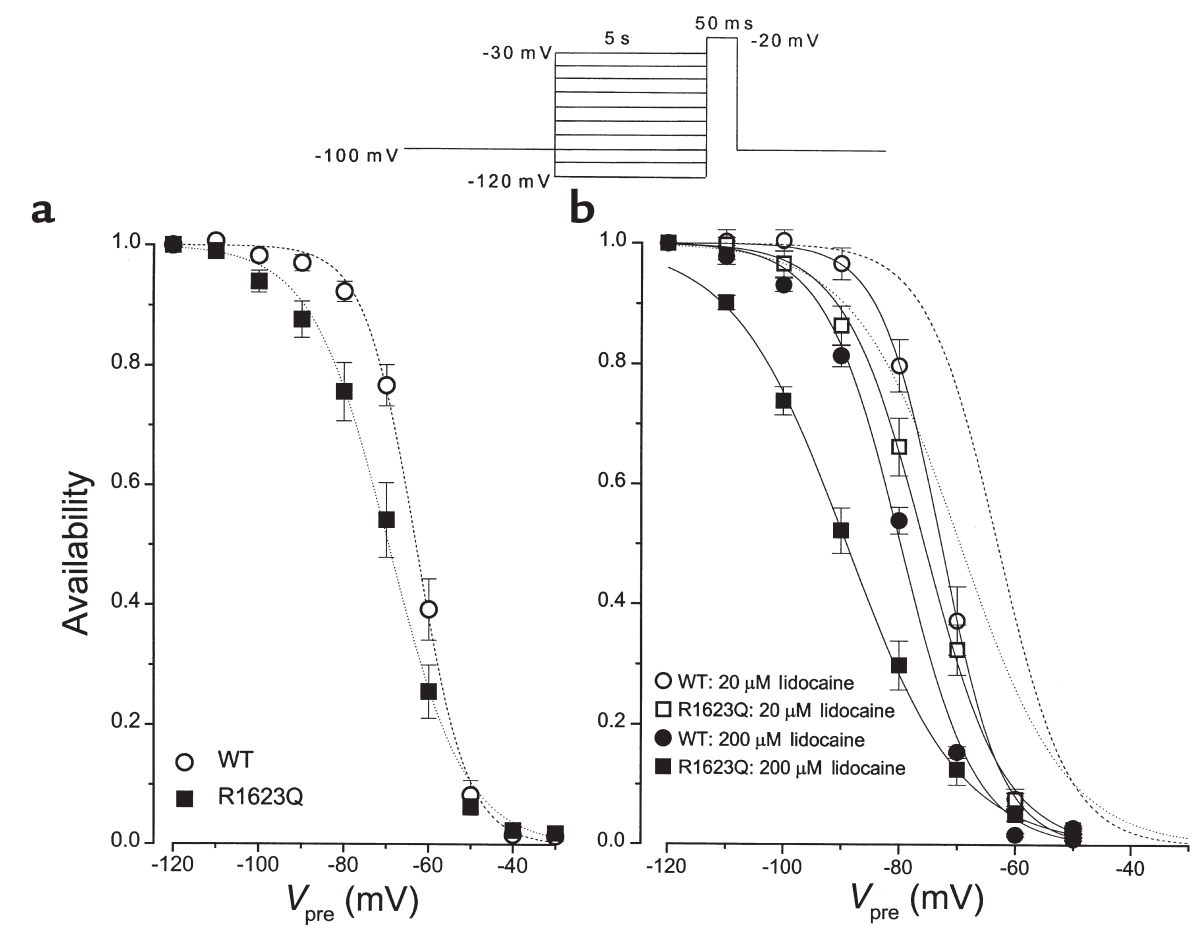

Figure 3

Voltage-dependent availability of $\mathrm{Na}$ channels. HEK cells stably expressing either wild-type or mutant Na channels were subjected to the voltage-clamp protocol shown (inset). (a) Peak inward $I_{\mathrm{Na}}$ at $-20 \mathrm{mV}$ is plotted as a function of the conditioning voltage for wild-type $(n=13)$ and R1623Q $(n=12)$, normalized to the peak current measured from $-120 \mathrm{mV}$. The solid curves represent nonlinear least-squares fits of the Boltzmann function $I / I_{\max }=\left(1+\exp \left[V-V_{1 / 2}\right] / k\right)^{-1}$ to the mean data, where $V_{1 / 2}$ is the membrane potential where channel availability is reduced by $50 \%$ and $k$ is the slope factor. Parameters obtained by fitting individual experiments are given in the text. (b) Lidocaine effect on $\mathrm{Na}$ channel availability. HEK cells expressing either WT ( $\mathrm{hH} 1$, circles) or mutant (R1623Q, squares) Na channels were subjected to the same voltage-clamp protocol in the presence of $20 \mu \mathrm{M}$ (open symbols) or $200 \mu \mathrm{M}$ (filled symbols) lidocaine. Solid lines show least-squares fits of a Boltzmann function to the mean data (see text for parameters from individual experiments). Dotted and dashed lines show the drugfree Boltzmann fits to the R1623Q and wild-type data (from a) for comparison. 


\section{Figure 4}

Time-dependent recovery of availability at $-80 \mathrm{mV}$. HEK cells expressing either WT (a)or mutant (b) Na channels were subjected to the voltageclamp protocol shown in the inset in the presence or absence of lidocaine. Data are plotted on a logarithmic scale to highlight the individual kinetic components of recovery. Fractional recovery was assessed as the ratio of peak $/_{\mathrm{Na}}$ during the second, relative to first, pulse (P2/P1) and is normalized to the 1,000-millisecond data point to eliminate differences in tonic block at $-80 \mathrm{mV}$ (per Figure $3 \mathrm{~b}$ ). The mean data were fitted to the biexponential function $y=A_{1}\left(1-e^{-t / \tau 1}\right)+A_{2}\left(1-e^{-t / \tau 2}\right)$, where $A_{1}, \tau_{1}$ are the amplitude and time constant of the prominent, rapid recovery component, and $A_{2}, \tau_{2}$ reflect a smaller, slow-recovery component. Fitted parameters were as follows: $\mathrm{WT}(n=8): A_{1}=0.79, \tau_{1}=31 \mathrm{~ms}, A_{2}=$ $0.21, \tau_{2}=169 \mathrm{~ms}$; WT + lidocaine $(n=8): A_{1}=0.80, \tau_{1}=23 \mathrm{~ms}, A_{2}=$ $0.20, \tau_{2}=85 \mathrm{~ms} ; \operatorname{R} 1623 \mathrm{Q}(n=12): A_{1}=0.82, \tau_{1}=9 \mathrm{~ms}, A_{2}=0.18, \tau_{2}=$ $100 \mathrm{~ms} ; \mathrm{R} 1623 \mathrm{Q}$ + lidocaine $(n=4): A_{1}=0.64, \tau_{1}=14 \mathrm{~ms}, A_{2}=0.36, \tau_{2}$ $=128 \mathrm{~ms}$. Lidocaine delayed the recovery of R1623Q channels, but did not slow wild-type recovery.

solid lines). Table 1 provides fitted parameters derived from the open-time distributions from a number of cell-attached patches. The R1623Q mutation increased the duration of the longer open-time component $\left(\tau_{2}\right)$ from 1.95 to 3.24 milliseconds $(P<0.05)$, suggesting a reduced rate of open-state inactivation, an effect consistent with the slowed rate of macroscopic current decay (in addition to more frequent reopenings). The mutation did not significantly change the relative proportion of short and long openings $\left(A_{1} / A_{2}\right)$, and the duration of the brief openings even slightly decreased $\left(\tau_{1}\right)$. Importantly, lidocaine did not change any of the open-time kinetic parameters for either the wild-type or R1623Q channel (Table 1). These results suggest that lidocaine does not directly block either the wild-type or mutant open channel with high (micromolar) affinity, nor does it speed or

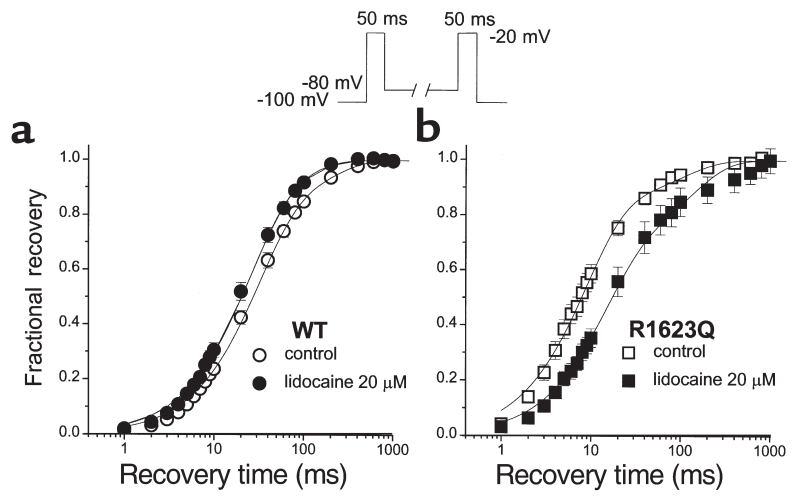

repair open-channel inactivation.

Conversely, inspection of the R1623Q unitary current records suggests that lidocaine did suppress the number of late openings, an effect consistent with the druginduced acceleration of whole-cell current decay (Figures 1 and 2). For R1623Q patches held at $-100 \mathrm{mV}$ before depolarization, $200 \mu \mathrm{M}$ lidocaine decreased the number of openings per sweep (corrected for channel number) from $1.36 \pm 0.09(n=6)$ to $0.61 \pm 0.20(n=4, P=0.004)$. We also assembled ensemble-average records from cells expressing either wild-type or R1623Q channels (Figure 6a) to determine the effect of lidocaine on the peak probability of channel opening (peak $\mathrm{P}_{\text {open }}$ ). There were 2 notable differences between the wild-type and R1623Q data. First, from a holding potential of $-120 \mathrm{mV}$, the drug-free peak probability of channel opening was far greater for R1623Q than wild-type (see also summary

\section{Figure 5}

Unitary currents from stably transfected HEK cells. Cell-attached patches were repeatedly depolarized to $-40 \mathrm{mV}$ for 150 milliseconds at either 10 -second (no drug) or 20 -second (200 $\mu \mathrm{M}$ lidocaine) intervals (holding potential, -100 or $-110 \mathrm{mV}$ ). Sweeps with channel openings were selected to emphasize the kinetic character of openings and reopenings and do not reflect the overall probability of opening in the conditions indicated. The patches shown contained $\leq 4$ active channels. Shown beneath each column of single-channel records are representative open-time histograms recorded under the same conditions. Each histogram includes data from 150-200 depolarizing sweeps. The histogram ordinates were normalized for visual comparison, and an exponential function $\left(y=A_{1} e^{-\mathrm{t} / \tau 1}+A_{2} e^{-\mathrm{t} / \tau 2}\right)$ was fitted to the data (solid line) to determine the dwell times. Parameters derived from histograms synthesized from individual patches are summarized in Table 1.

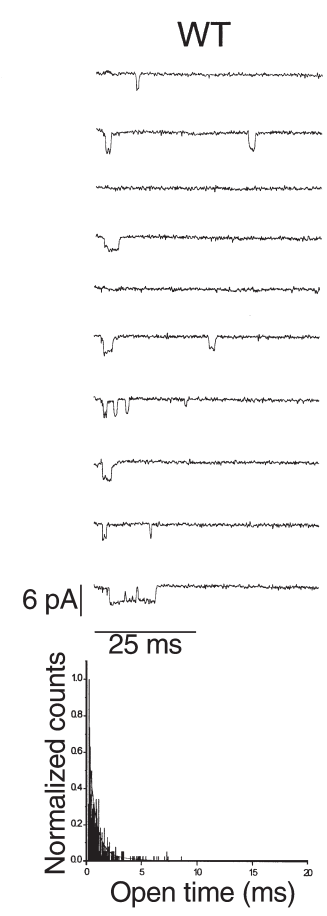

$\mathrm{WT}+200 \mu \mathrm{M}$ LIDO
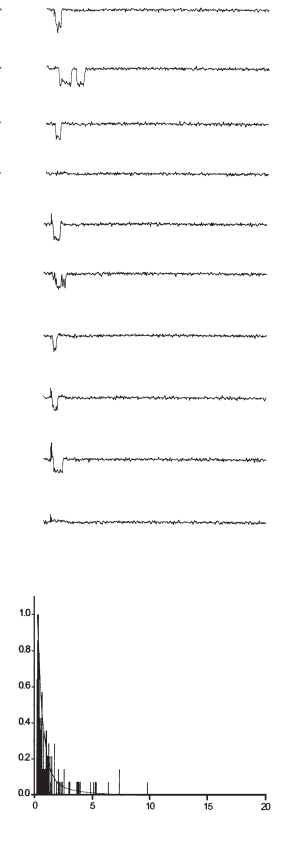
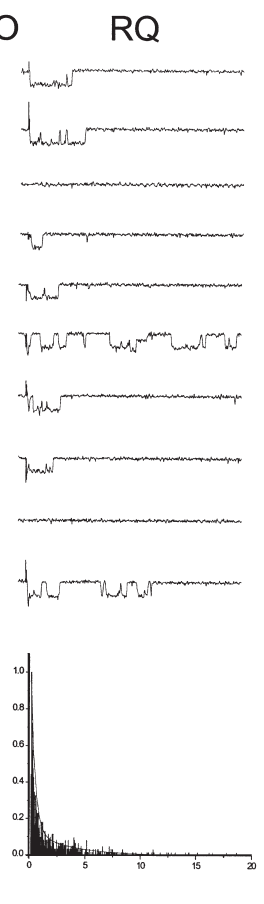
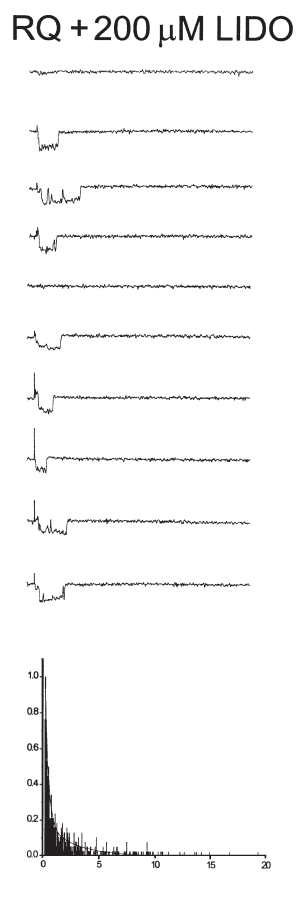
data from a number of patches, Figure 6b). Figure 3a clarifies that both wild-type and R1623Q drug-free channels are fully available at $-120 \mathrm{mV}$. Hence, this drug-free difference in the peak probability of channel opening does not arise from differences in closed-state inactivation, but may result from the longer open time for $\mathrm{R} 1623 \mathrm{Q}$, which increases the likelihood that first openings will occur "in register". Second, $200 \mu \mathrm{M}$ lidocaine had little to no effect on the peak $\mathrm{P}_{\text {open }}$ of wild-type channels, but markedly reduced the peak $\mathrm{P}_{\text {open }}$ of the $\mathrm{R} 1623 \mathrm{Q}$ mutant, consistent with the increased tonic-block sensitivity of whole-cell R1623Q current compared with wild-type in Figure 1. A

a

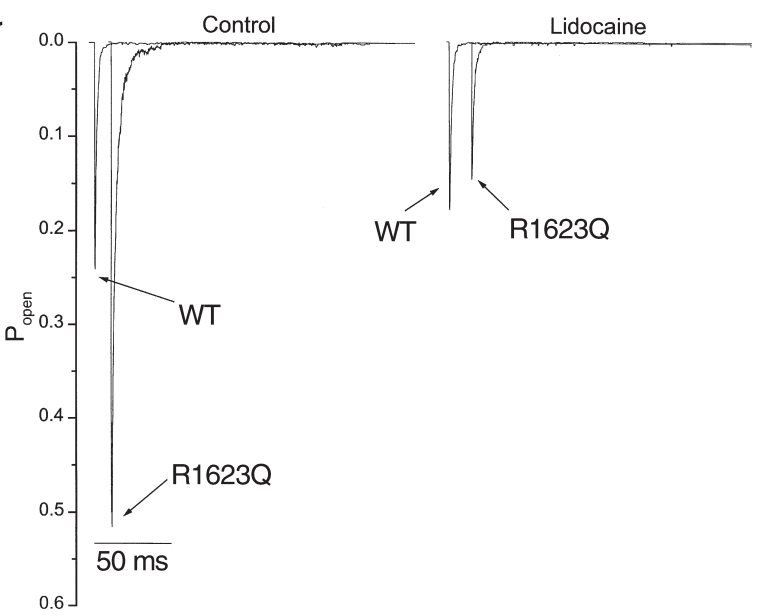

b

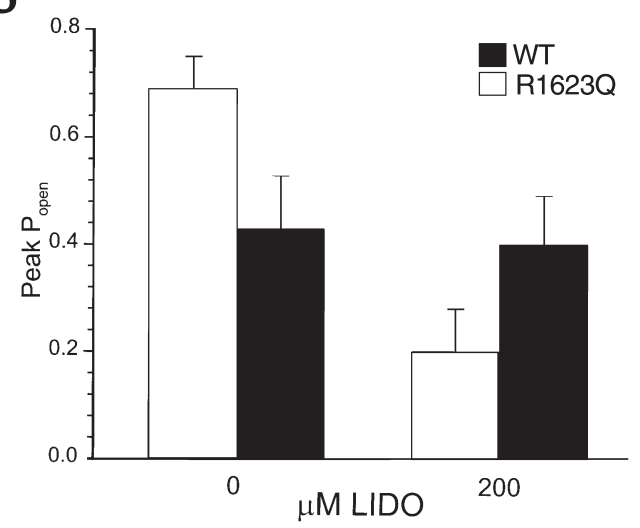

Figure 6

Ensemble-average currents and lidocaine. (a) Ensemble-average currents derived multichannel patches containing wild-type or R1623Q channels in the absence (left) and presence (right) of $200 \mu \mathrm{M}$ lidocaine. Representative wild-type and R1623Q ensembles are shown superimposed, but shifted on the time axis, to facilitate comparison. Patches were held at $-120 \mathrm{mV}$ for 5 seconds and then pulsed to -40 $\mathrm{mV}$ for 150 milliseconds at a repetition cycle of either 10 seconds (no drug) or 20 seconds (drug). Approximately 150 consecutive sweeps for each patch were then used to construct $P_{\text {open }}$ ensembles that are corrected for the number of channels in the patch and the unitary current amplitude ( 2.5 pA). (b) Data summarizing the peak probability of channel opening in the presence and absence of $200 \mu \mathrm{M}$ lidocaine (number of patches: wild-type, $n=3$; R1623Q, $n=3$; wildtype + lidocaine, $n=3$; R1623Q + lidocaine, $n=5$ ). unifying mechanism that rationalizes the increased lidocaine effect on R1623Q peak $I_{\mathrm{Na}}$ (peak-opening probability) and macroscopic current decay rate (probability of late openings) is considered next.

\section{Discussion}

A domain IV-S4 mutation modifies inactivation and angments lidocaine sensitivity. Single-channel analysis (Figure 5) of the R1623Q gating phenotype reveals impaired open-state inactivation consistent with the slowed rate of whole-cell $\mathrm{I}_{\mathrm{Na}}$ decay (Figures 1, 2, and 6), consistent with previous reports $(22,23)$. In addition, the voltage-dependent availability of $I_{\mathrm{Na}}$ is shifted to more negative membrane potentials (Figure 3 ), indicating that inactivation from closed states is increased. A slowing of open-state inactivation with coexistent enhancement of closed-state inactivation for this hH1 mutant is consistent with gating phenotypes reported for mutations in the domain IV, S4 segment of the human skeletal muscle Na channel (hSkM1) linked to paramyotonia congenita $(24,33)$.

When exposed to lidocaine, R1623Q peak $I_{\mathrm{Na}}$ is blocked more potently than wild-type hH1 in both whole-cell (Figure 1) and single-channel ensembleaverage recordings (Figure 6), a pharmacologic phenotype shared by the analogous domain IV, S4 paramyotonia mutant in hSkM1 (R1448C) (20). In addition, we find that the rate of R1623Q $I_{\mathrm{Na}}$ decay is speeded to a much greater extent than wild-type when measured in whole-cell recordings from oocytes or HEK cells (Figures 1 and 2) or from ensemble-averaged unitary currents (Figure 6). This enhanced drug sensitivity could result if the mutation increased the affinity of the open state for lidocaine, or, alternatively, the open-state inactivation mechanism could be sensitive to lidocaine (22). Figure $2 \mathrm{~b}$ shows that the effect of lidocaine on the rate of R1623Q $I_{\mathrm{Na}}$ decay was largely voltage independent from -30 to $+20 \mathrm{mV}$, whereas the rate of cardiac $\mathrm{Na}$ channel open-state inactivation increases substantially over this membrane potential range $(32,34)$. Moreover, Figure 5 indicates that a high lidocaine concentration $(200 \mu \mathrm{M})$ that markedly speeded the rate of $I_{\mathrm{Na}}$ decay and reduced the number of openings per sweep had no effect on the mean open time. Taken together, these findings suggest that the enhanced lidocaine sensitivity of R1623Q is not linked to an increase in open-state block nor to an enhanced rate of open-state inactivation.

Because lidocaine does not appear to influence inactivation from open states, we considered the possibility that increased closed-state inactivation may underlie the increased drug sensitivity of the mutant. In support of this notion, our results clarify that mutant channels held at membrane potentials near $V_{\text {rest }}$ are more susceptible to closed-state inactivation (Figure 3a), and R1623Q channels exposed to lidocaine at these membrane potentials are more susceptible to block than wild-type (Figure 3b). During depolarization sufficient to open channels, a proportion of channels inac- 


\section{Figure 7}

Three-state Markov gating models with state-dependent lidocaine binding. The symbols $\mathrm{C}, \mathrm{O}$, and I represent the aggregate closed, open, and inactivated states of the channel, respectively. (a) Oneaffinity (model I) and 2-affinity (model II) schemes of lidocaine binding were assessed. For the wild-type channel, the rate constants used for the gating transitions upon depolarization were as follows $\left(\mathrm{s}^{-1}\right): k_{\mathrm{CO}}$ (that is, closed $\rightarrow$ open $)=665, k_{\mathrm{OC}}=492, k_{\mathrm{OI}}$ $=1998, k_{1 \mathrm{O}}=2.9, k_{\mathrm{Cl}}=66$. The magnitude of $k_{\mathrm{IC}}$ was determined by microscopic reversibility constraints: $k_{\mathrm{IC}}=k_{\mathrm{OC}} k_{\mathrm{CI}} k_{\mathrm{IO}} /\left(k_{\mathrm{OC}} k_{\mathrm{OI}}\right)$. For R1623Q, differences from wild-type were $k_{\mathrm{OI}}=658$ and $k_{\mathrm{CI}}=$ 492. For LQT3, the only difference from wild-type was $k_{1 \mathrm{O}}=55$. For models I and II, the on-rate constants for drug binding were always $1 \times 10^{8} \mathrm{~s}^{-1} \mathrm{M}^{-1}$. In model II, the off-rate constant for lidocaine binding to the $C$ state $\left(k_{\text {Coff }}\right)$ was $128,027 \mathrm{~s}^{-1}\left(K_{d} 1.3 \mathrm{mM}\right)$. In models I and II, the off-rate constant for lidocaine binding to the I state $\left(k_{\text {loff }}\right)$ was $383 \mathrm{~s}^{-1}\left(K_{\mathrm{d}} 4 \mu \mathrm{M}\right)$. In both models, we assumed that lidocaine binding did not slow the rate constant for recovery from inactivation as recently shown (38). Hence, $k_{\mathrm{IL} \rightarrow \mathrm{CL}}$ was equal to $k_{\mathrm{IC}}$. The forward rate constant for closed-state inactivation when lidocaine is bound $\left(k_{\mathrm{CL} \rightarrow \mathrm{IL}}\right)$ was entirely determined (through microscopic reversibility) by the off rates for drug binding to the closed and inactivated states and the drug-free rate of closed-state inactivation as follows: $\mathrm{k}_{\mathrm{CL} \rightarrow \mathrm{IL}}=k_{\mathrm{Cl}} k_{\mathrm{Coff}} / k_{\text {loff. }}$. Because $k_{\text {loff }}<k_{\text {Coff }}$, the rate of closed-state inactivation is increased when drug is bound (i.e., $k_{\mathrm{CL} \rightarrow \mathrm{LL}}>k_{\mathrm{CI}}$ ). (b) Simulated sodium currents from models I and II (see text).

tivate directly from closed states without opening (35, 36). If lidocaine block is somehow augmented by closed-state inactivation, then enhanced lidocaine block of the R1623Q mutant could develop rapidly during depolarization.

Could an interaction between lidocaine and closedstate inactivation upon depolarization explain the enhanced block of R1623Q peak current as well as the drug-induced speeding of $I_{\mathrm{Na}}$ decay? Because closed, inactivated, and drug-bound states are all nonconducting, the interplay between closed-state inactivation and drug binding at membrane potentials sufficient to open the channel is difficult to assess directly. Hence, to examine the feasibility of this mechanism, we performed quantitative simulations using a simplified $\mathrm{Na}$ channel gating scheme (Figure 7a) that recapitulates a general feature of contemporary cardiac $\mathrm{Na}$ channel gating models (37): channels could either open before inactivating or could inactivate directly from the closed state. The drug-free R1623Q phenotype was simulated by a reduction in the rate of open-state inactivation, as described previously (22), plus an increase in the rate of closed-state inactivation (Figure 7). We first assessed whether drug binding to the inactivated state alone (regardless of the pathway to inactivation) could account simultaneously for lidocaine effects on peak $I_{\mathrm{Na}}$ and $I_{\mathrm{Na}}$ decay in R1623Q (Figure 7a, model I). Despite rapid, high-affinity binding to the inactivated state $\left(K_{\mathrm{d}}\right.$ $=4 \mu \mathrm{M}$, on rate for drug binding was diffusion limited at $\left.10^{8} \mathrm{~s}^{-1} \mathrm{M}^{-1}\right), 20 \mu \mathrm{M}$ lidocaine had a negligible effect on the current (Figure 7b), as did $200 \mu \mathrm{M}$ (not shown).

Surprisingly, adding low-affinity $(\sim \mathrm{mM})$ lidocaine a

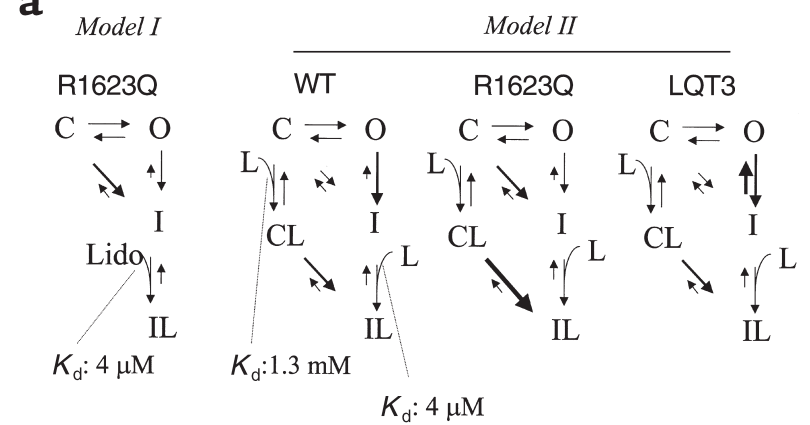

b
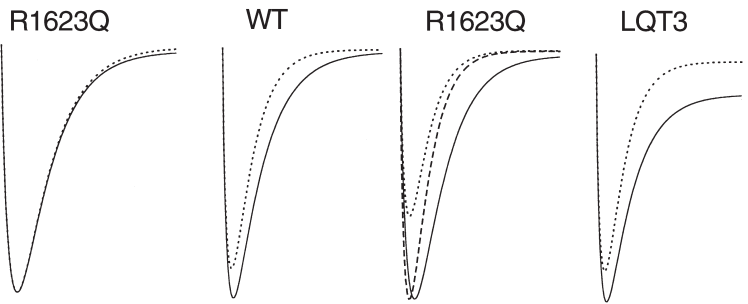

$\overline{2 \mathrm{~ms}}$

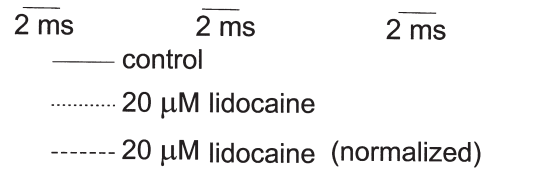

binding (i.e., tonic block) to the closed, noninactivated channel (Figure 7a, model II) substantially altered the response of the model to low $(\sim \mu \mathrm{M})$ drug concentrations (Figure $7 \mathrm{~b}$ ). Given the assumption that the affinity of the inactivated channel for lidocaine exceeds that of the closed channel and assuming that closed drugbound channels do inactivate, microscopic reversibility constraints dictate that the likelihood of closed-state inactivation is increased by drug binding (see legend Figure 7 for explicit formulation). Under these conditions, the simulation indicates that $20 \mu \mathrm{M}$ lidocaine was sufficient to markedly reduce the R1623Q $I_{\mathrm{Na}}$ peak and also speed the rate of current decay (Figure $7 \mathrm{~b}$, model II middle, dotted line). The dashed line (Figure $7 \mathrm{~b}$, model II middle) shows the drug-modified current normalized to the control (drug free) current to emphasize the effect of lidocaine on the rate of current decay. In contrast, the same concentration of lidocaine minimally changed either characteristic of the wild-type current (model II, left), as seen experimentally (Figures 1 and 2).

We considered whether this mechanism could apply generally to autosomal dominant LQT3 SCN5A mutations. The gating consequences of these mutations dif-

Table 1

Open time distributions

\begin{tabular}{lrrr}
\hline & \multicolumn{1}{c}{$\tau_{1}(\mathrm{~ms})$} & \multicolumn{1}{c}{$\tau_{2}(\mathrm{~ms})$} & \multicolumn{1}{c}{$A_{1} / A_{2}$} \\
WT $(n=4)$ & $0.53 \pm 0.06$ & $1.95 \pm 0.44$ & $11.24 \pm 0.74$ \\
WT + lidocaine $(n=3)$ & $0.41 \pm 0.04$ & $1.47 \pm 0.58$ & $18.63 \pm 3.62$ \\
RQ $(n=11)$ & ${ }^{A} 0.39 \pm 0.02$ & ${ }^{A} 3.24 \pm 0.30$ & $15.34 \pm 3.60$ \\
RQ + lidocaine $(n=9)$ & $0.34 \pm 0.02$ & $2.75 \pm 0.27$ & $23.28 \pm 10.87$
\end{tabular}

ADiffers from drug-free wild-type value, $P<0.05$. 
fer from R1623Q: these mutations generally do not slow the early rate of $I_{\mathrm{Na}}$ decay, but consistently produce a plateau of noninactivating current, suggesting the inactivated state is rendered nonabsorbing by the mutation $(9,10)$. This LQT3 macroscopic gating phenotype can be reproduced empirically by increasing the rate constant of recovery from the inactivated to the open state (22, 37). Model II (Figure 7b, right) shows LQT3 Na currents simulated by this approach, with preserved wild-type closed-state inactivation gating kinetics. Notably, the LQT3 current decay rate was similar to wild-type (unlike R1623Q), but the increased rate constant for recovery from open-state inactivation imparted a plateau of noninactivating current. In our simulation, lidocaine binding had a small effect on the LQT3 $I_{\text {Na }}$ peak (dotted line), similar to that of wild-type (Model II, left), but nearly eliminated the plateau of noninactivating current. This result recapitulates the experimental findings for lidocaine block of $\mathrm{Na}$ channels modified by the long QT $\triangle$ KPQ 1505-1507 deletion mutation (Figure $4 \mathrm{c}$ in ref. 16). A greater drug sensitivity of the noninactivating current plateau relative to peak $I_{\mathrm{Na}}$ has also been demonstrated for the lidocaine analogue, mexiletine, in 3 LQT3 mutations ( $\triangle \mathrm{KPQ}, \mathrm{N} 1325 \mathrm{~S}$, and R1644H) (18). We conclude that potentiation of an altered closed-state inactivation gating mechanism accounts for the unique effects of lidocaine on the R1623Q mutant. Moreover, the modeling analysis suggests that the same drug effect on unmodified closed-state inactivation gating could underlie the more general salutary effects of $\mathrm{Na}$ channel-blocking agents in other long QT disorders.

\section{Acknowledgments}

This work was supported by the National Institutes of Health (R01 GM-56307 to J.R. Balser, R01 HL-52668 to E. Marbán, R01 HL50411 to G.F. Tomaselli). We thank Dan Roden and Jean-Francois Desaphy for helpful discussion and review of the manuscript.

1. Shimizu, W., and Antzelevitch, C. 1997. Sodium channel block with mexiletine is effective in reducing dispersion of repolarization and preventing torsade de pointes in LQT2 and LQT3 models of the long-QT syndrome. Circulation. 96:2038-2047.

2. Stühmer, W., et al. 1989. Structural parts involved in activation and inactivation of the sodium channel. Nature. 339:597-603.

3. West, J., et al. 1992. A cluster of hydrophobic amino acid residues required for fast $\mathrm{Na}^{+}$-channel inactivation. Proc. Natl. Acad. Sci. USA. 89:10910-10914.

4. Smith, M.R., and Goldin, A.L. 1997. Interaction between the sodium channel inactivation linker and domain III S4-S5. Biophys, J. 73:1885-1895.

5. McPhee, J.C., Ragsdale, D.S., Scheuer, T., and Catterall, W.A. 1998. A critical role for the intracellular loop in domain IV of the sodium channel $\alpha$ subunit in fast inactivation. J. Biol. Chem. 273:1121-1129.

6. Lerche, H., et al. 1997. Role in fast inactivation of the IV/S4-S5 loop of the human muscle $\mathrm{Na}^{+}$channel probed by cysteine mutagenesis. J. Physiol. (Lond). 505:345-52.

7. Filatov, G.N., Nguyen, T.P., Kraner, S.D., and Barchi, R.L. 1998. Inactivation and secondary structure in the D4/S4-5 region of the SkM1 sodium channel. J. Gen. Physiol. 111:703-15.

8. Tang, L., Chehab, N., Wieland, S.J., and Kallen, R.G. 1998. Glutamine substitution at alanine 1649 in the S4-S5 cytoplasmic loop of domain 4 removes the voltage sensitivity of fast inactivation in the human heart sodium channel. J. Gen. Physiol. 111:639-52.

9. Bennett, P.B., Yazawa, K., Naomasa, M., and George, A.L. 1995. Molecular mechanism for an inherited cardiac arrhythmia. Nature. 376:683-685.

10. Dumaine, R., et al. 1996. Multiple mechanisms of $\mathrm{Na}^{+}$channel-linked longQT syndrome. Circ. Res. 78:916-924.
11. Wang, D.W., Yazawa, K., George, A.L., and Bennett, P.B. 1996. Characterization of human cardiac $\mathrm{Na}^{+}$channel mutations in the congenital long QT syndrome. Proc. Natl. Acad. Sci. USA. 93:13200-13205.

12. Ragsdale, D.S., McPhee, J.C., Scheuer, T., and Catterall, W.A. 1994. Molecular determinants of state-dependent block of $\mathrm{Na}^{+}$channels by local anesthetics. Science. 265:1724-1728.

13. Ragsdale, D.S., McPhee, J.C., Scheuer, T., and Catterall, W.A. 1996. Common molecular determinants of local anesthetic, antiarrhythmic, and anticonvulsant block of voltage-gated $\mathrm{Na}^{+}$channels. Proc. Natl. Acad. Sci. USA. 93:9270-9275.

14. Sunami, A., Dudley, S.C., Jr., and Fozzard, H.A. 1997. Sodium channel selectivity filter regulates antiarrhythmic drug binding. Proc. Natl. Acad. Sci. USA. 94:14126-31.

15. Bennett, P.B., Valenzuela, C., Li-Qiong, C., and Kallen, R.G. 1995. On the molecular nature of the lidocaine receptor of cardiac $\mathrm{Na}^{+}$channels. Circ. Res. 77:584-592.

16. An, R.H., Bangalore, R., Rosero, S.Z., and Kass, R.S. 1996. Lidocaine block of LQT-3 mutant human Na channels. Circ. Res. 79:103-108.

17. Schwartz, P.J., et al. 1995. Long QT syndrome patients with mutations of the SCN5A and HERG genes have differential responses to $\mathrm{Na}^{+}$channel blockade and to increases in heart rate. Implications for gene-specific therapy. Circulation. 92:3381-6.

18. Wang, D.W., Yazawa, K., Makita, N., George, A.L., and Bennett, P.B. 1997. Pharmacological targeting of long QT mutant sodium channels. J. Clin. Invest. 99:1714-1720.

19. Balser,J.R, et al. 1996. Local anesthetics as effectors of allosteric gating: lidocaine effects on inactivation-deficient rat skeletal muscle Na channels. J. Clin. Invest. 98:2874-2886

20. Fan, Z., George, A.L., Kyle, J.W., and Makielski, J.C. 1996. Two human paramyotonia congenita mutations have opposite effects on lidocaine block of $\mathrm{Na}^{+}$channels expressed in a mammalian cell line. J. Physiol. 496:275-286.

21. Yamagishi, H., et al. 1998. A de novo missense mutation (R1623Q) of the SCN5A gene in a Japanese girl with sporadic long QT syndrome. Mutations in brief no. 140. Hum. Mutat. 11:481.

22. Kambouris, N.G., et al. 1998. Phenotypic characterization of a novel long QT syndrome mutation in the cardiac sodium channel. Circulation. 97:640-644.

23. Makita, N., et al. 1998. A de novo missense mutation of human cardiac $\mathrm{Na}^{+}$ channel exhibiting novel molecular mechanisms of long QT syndrome. FEBS Lett. 423:5-9.

24. Chahine, M., et al. 1994. Sodium channel mutations in paramyotonia congenita uncouple inactivation from activation. Neuron. 12:281-294.

25. Chen, L.Q., Santarelli, V., Horn, R., and Kallen, R.G. 1996. A unique role for the 44 segment of domain 4 in the inactivation of Na channels. J. Gen. Physiol. 108:549-556.

26. Weiner, M.P., Felts, K.A., Simcox, T.G., and Brama, J.C. 1993. A method for the site-directed mono- and multi-mutagenesis of double stranded DNA. Gene. 126:35-41.

27. Johns, D.C., Nuss, H.B., and Marban, E. 1997. Suppression of neuronal and cardiac transient outward currents by viral gene transfer of dominant negative KV4.2 constructs. J. Biol. Chem. 272:31598-31603.

28. Hamill, O.P., Marty, A., Neher, E., Sakmann, B., and Sigworth, F.J. 1981 Improved patch-clamp techniques for high-resolution current recording form cells and cell-free membrane patches. Pflügers Arch. 391:85-100.

29. Hindmarsh, A.C. 1983. Odepack, a systematized collection of ode solvers. In Scientific computing. R.S. Stepleman, editor. North-Holland Company, Amsterdam, The Netherlands. 55-64.

30. Petzold, L.R. 1983. Automatic selection of methods for solving stiff and nonstiff systems of ordinary differential equations. Society for Industrial and Applied Mathematics Journal on Scientific and Statistical Computing. 4:136-148.

31. Balser, J.R., Roden, D.M., and Bennett, P.B. 1990. Global parameter optimization for cardiac potassium channel gating models. Biophys. J. 57:433-444

32. Lawrence, J.H., Yue, D.T., Rose, W.C., and Marban, E. 1991. Sodium channel inactivation from resting states in guinea-pig ventricular myocytes. J. Physiol. 443:629-650

33. Ji, S., George, A.L., Horn, R., and Barchi, R.L. 1996. Paramyotonia congenita mutations reveal different roles for segments S3 and S4 of domain D4 in hSkM1 sodium channel gating. J. Gen. Physiol. 107:183-194.

34. Yue, D.T., Lawrence, J.H., and Marban, E. 1989. Two molecular transitions influence cardiac sodium channel gating. Science. 244:349-352.

35. Horn, R., Patlak, J.B., and Stevens, C.F. 1981. Sodium channels need not open before they inactivate. Nature. 291:426-427.

36. Scanley, B.E., Hanck, D.A., Chay, T., and Fozzard, H.A. 1990. Kinetic analysis of single sodium channels from canine cardiac Purkinje cells. J. Gen. Physiol. 95:411-437.

37. Clancy, C.E., and Rudy, Y. 1999. Linking a genetic defect to its cellular phenotype in a cardiac arrhythmia. Nature. 400:566-9.

38. Vedantham, V., and Cannon, S.C. 1999. The position of the fast-inactivation gate during lidocaine block of voltage-gated $\mathrm{Na}^{+}$channels. J. Gen. Physiol. 113:7-16. 